\title{
Surgical Management of Benign Adnexal Masses in the Pediatric/Adolescent Population:
}

\section{An 11-Year Review}

Lauren M. Bergeron $\mathrm{MD}^{1}$, Katherine C. Bishop $\mathrm{MD}^{1}$, Holly R. Hoefgen MD ${ }^{1}$, Margaret S. Abraham MD ${ }^{1}$, Nhial T. Tutlam MPH ${ }^{1}$, Diane F. Merritt MD ${ }^{1}$, Jeffrey F. Peipert MD, $\mathrm{PhD}^{2}$ Department of Obstetrics and Gynecology, Washington University School of Medicine; Saint Louis, $\mathrm{MO}^{1}$ Department of Obstetrics and Gynecology, Indiana University School of Medicine; Indianapolis, Indiana ${ }^{2}$

Address for correspondence:

Lauren Bergeron, MD

Department of Obstetrics and Gynecology

Washington University School of Medicine

4911 Barnes Jewish Hospital Plaza, Campus Box 8064

St. Louis, MO 63110

Fax: 314-747-1490

Email: BergeronL@wudosis.wustl.edu

Financial Support: None

Disclaimers: Dr. Peipert receives research funding from Teva Pharmaceuticals Industries, Bayer Healthcare Pharmaceuticals, and Merck \& Co., Inc., and serves on advisory boards for Teva Pharmaceutical Industries and Perrigo

This is the author's manuscript of the article published in final edited form as:

Bergeron, L. M., Bishop, K. C., Hoefgen, H. R., Abraham, M. S., Tutlam, N. T., Merritt, D. F., \& Peipert, J. F. (2017). Surgical management of benign adnexal masses in the pediatric/adolescent population: an 11-year review. Journal of pediatric and adolescent gynecology, 30(1), 123-127. https://doi.org/10.1016/j.jpag.2016.09.002 
34

35

36

37

\section{$\underline{\text { Abstract }}$}

Study Objective: The purpose of this study was to compare ovarian conservation rates and surgical approach in benign adnexal surgeries performed by surgeons versus gynecologists at a tertiary care institution.

Design: A retrospective cohort review.

Setting: Children's and Adult tertiary care university-based hospital.

Participants: Patients $\leq 21$ years of age undergoing surgery for an adnexal mass from January 2003 through December 2013.

Interventions: Patient age, demographics, menarchal status, clinical symptoms, radiologic imaging, timing of surgery, surgeon specialty, mode of surgery, rate of ovarian conservation and pathology were recorded. Patients were excluded if they had a uterine anomaly or pathologyproven malignancy.

Main outcome measures: The primary outcome was the rate of ovarian conservation relative to surgical specialty; secondary outcome was surgical approach relative to surgical specialty. Results: Of 310 potential cases, 194 met inclusion criteria. Gynecologists were more likely than surgeons to conserve the ovary $(80$ vs. $63 \%$; odds ratio [OR] 2.28 ; $95 \%$ confidence interval [CI] 1.16-4.48). After adjusting for age, body mass index, mass size and urgency of surgery, the difference was attenuated (adjusted OR 1.84; CI 0.88-3.84). Surgeons and gynecologists performed minimally invasive surgery at similar rates, $62 \%$ vs. $50 \%(\mathrm{P}=0.11)$. A patient was more likely to be operated on by a gynecologist if she was older $(\mathrm{P}<0.001)$ and post-menarchal $(\mathrm{P}=0.005)$.

Conclusion: Our study suggests that gynecologists are more likely to perform ovarian-conserving surgery. However, our sample size precluded precise estimates in our multivariable model. 
57 Educational efforts among all pediatric and gynecologic surgeons should emphasize ovarian

58 conservation and fertility preservation whenever possible.

59 Key words: Pediatric, adolescent, adnexal mass, adnexal torsion, ovarian conservation,

60 laparoscopy

61

62

63

64

65

66

67

68

69

70

71

72

73

74

75

76

77

78

79 


\section{$\underline{\text { Introduction }}$}

The estimated incidence of adnexal masses is approximately 2.6 cases in 100,000 girls in childhood; rates in adolescents are suspected to be higher, but precise population-based estimates $\operatorname{are~lacking~}^{1}$. The majority of adnexal masses are benign; the incidence of malignancy ranges between $4 \%$ and $11 \%$ for all surgically excised adnexal neoplasms in children and adolescents ${ }^{2-4}$. Patients are risk stratified according to mass size and characteristics. Masses that appear benign and are less than $8 \mathrm{~cm}$ in maximum diameter are often managed conservatively unless symptoms determine the need for surgery $y^{5,6}$. When operative intervention is indicated, it can be performed by laparotomy or laparoscopy with treatment ranging from simple cyst aspiration to salpingooophorectomy. Adnexal surgeries are performed by a number of surgical specialists including pediatric surgeons, general surgeons, gynecologists, and gynecologic-oncologists.

Patterns in surgical care have evolved, and the paradigm has shifted from ovarian removal to ovarian evaluation and conservation. However, this shift has occurred more slowly in girls and adolescents than in adult women ${ }^{3}$. This is concerning because after unilateral oophorectomy, the possibility of surgical castration prior to the completion of reproduction increases should the contralateral ovary become affected by torsion or neoplasia ${ }^{7}$. Thus, it is important to perform fertility-preserving surgery when feasible.

Over the past decade, minimally invasive surgical techniques have become the standard of care for removing benign adnexal masses because of shorter recovery time, decreased pain, and improved cosmesis ${ }^{3,8-10}$. Rogers et al. concluded that it is safe in children and adolescents to proceed with a laparoscopic approach for adnexal masses without complex features measuring less than or equal to $8 \mathrm{~cm}$ in maximum diameter ${ }^{5}$. In 2012, Berger-Chen et al. conducted a population-based analysis to determine factors associated with the performance of ovarian- 
103

conserving cystectomy and minimally invasive surgery in adolescents with benign ovarian masses. They found that physician, hospital, and patient characteristics all strongly influenced treatment ${ }^{3}$. Additional studies have shown that gynecologists are more likely than other surgeons to perform these surgeries in a minimally-invasive fashion and conserve the ovary ${ }^{2-4,7}$.

Ovarian-conserving procedures has proven safe for adolescents and over the last decade minimally invasive surgical techniques have become the gold- standard treatment ${ }^{1-4,8-13}$. Prior studies have already shown that gynecologists are more likely than surgeons to conserve the ovary $^{2-4}$. The purpose of this study was to review all cases of adnexal masses treated at our institution (Saint Louis Children's Hospital and Barnes-Jewish Hospital) to determine whether ovarian-conserving cystectomy and minimally invasive surgery rates in young girls and adolescents differed between surgeons and gynecologists. More specifically, we hoped to determine the factors associated with ovarian conservation and a minimally invasive surgical approach. We also wanted to trend the rates of ovarian sparing and minimally invasive surgery between surgeons and gynecologists over time.

\section{Materials and Methods}

We performed a retrospective cohort study of patients treated for a benign adnexal mass at St. Louis Children's Hospital (SLCH) and Barnes Jewish Hospital (BJH), both affiliated hospitals of Washington University School of Medicine in St. Louis. Cases were collected over the 11-year period from January 2003 through December 2013. Before initiating this research, permission was obtained from the Institutional Review Board.

Patients were identified by using common International Classification of Diseases (ICD)9 codes for an adnexal mass $(620.2,620.5$ and 625.8) and Current Procedural Terminology 
(CPT) surgical codes (58660 - 58999). Cases were excluded if the patient: 1) was older than 21 years of age at the time of surgery; 2) was undergoing surgery for suspected ectopic pregnancy or pelvic inflammatory disease; 3) had uterine anomalies; 4) had pathology-proven malignancy; or 5) had incomplete medical records.

Data were extracted from electronic and paper hospital patient records. Information on patient demographics, menarchal status, clinical signs and symptoms, largest dimension of mass, timing of procedure, surgeon specialty, operative procedure, conversion of laparoscopic to laparotomy, specimen size and histologic diagnosis were all recorded.

Attending surgeon specialty was classified as gynecologist (all gynecologic specialties including oncology) or surgeon (general, pediatric, or other subspecialty). The entrance into the peritoneal cavity was recorded as laparoscopic if a minimally invasive approach was maintained throughout the entire case or laparotomy if the procedure was performed via open abdominal incision. Cases in which the surgeon performed a mini-laparotomy or converted a minimally invasive approach to an open abdominal procedure were recorded as laparotomy. Radiologic mass size was recorded as largest dimension in centimeters on ultrasound, computed tomography, or magnetic resonance imaging. If two or more modalities were utilized, the larger size was recorded. If preoperative size was not recorded, the mass size recorded in the surgeon's operative note was used. Cases were classified as "torsion only" in those that had no other histopathologic diagnosis. If a patient underwent two separate adnexal surgeries, each procedure was recorded separately. Timing of procedure was classified as emergent if surgery was performed within 24 hours of the physician's initial evaluation and non-emergent if performed after 24 hours or scheduled as outpatient. 
Characteristics of the study sample including demographics, menarchal status, mass size, rates of ovarian conservation, surgical approach, surgeon type, timing of surgery, and conversion rates were described using frequencies, percentage, means and standard deviations where appropriate. Between-group differences were analyzed by chi-square or Fisher exact tests for categorical variables, and ANOVA for normally distributed continuous variables. Logistic regression analysis was performed and odds ratios with $95 \%$ confidence intervals were calculated for ovarian conservation and surgical approach according to specialist. Confounding variables were identified by multivariate analysis and the odds ratios were adjusted for the confounding variables age, body mass index (BMI), largest mass size, and timing of surgery. Statistical Analysis Software (SAS) was used for statistical analyses and the significance level alpha was set at 0.05 .

\section{$\underline{\text { Results }}$}

A total of 310 charts were analyzed (277 charts from SLCH and 33 from BJH). Eightyone charts from SLCH and 6 charts from BJH were excluded because surgery did not involve the adnexa. Further cases were excluded for missing chart information $(n=11)$, uterine anomalies $(n=3)$, and pathology-proven malignancy $(n=15)$. In total, 191 pediatric and adolescent patients undergoing 194 procedures were included in this analysis (Figure 1). Analyses including and excluding the three repeat patients produced similar results, so the analyses presented here include 194 procedures.

The mean age of patients was 13, and the mean BMI was 26. Thirty-four percent of patients were African American and 65\% Caucasian. The majority (74\%) of patients were postmenarchal; however, menarchal status was unknown in $14 \%$ of our sample. The average size of the adnexal mass was $8.8 \mathrm{~cm}$. Gynecologists performed $38 \%$ of the cases. The majority of cases 
173

174

175

176

177

178

179

180

181

182

183

184

185

186

187

188

189

190

191

192

193

194

195
(69\%) were performed non-emergently, 57\% were performed laparoscopically, and $14 \%$

converted from laparoscopic to laparotomy.

The surgeon group performed 35 torsion cases and the gynecologist group performed 24 .

Physiologic cysts were two times more common in the surgeon group (50 vs. 24). There were 35 mature teratoma cases in the surgical group and 22 in the gynecology group. The numbers of other benign neoplasias, including serous and mucinous cystadenomas, fibromas, and endometriomas, were similar between the two groups (surgeons 17, gynecologists 14).

Table 1 shows patient demographics relative to ovarian conservation, surgical approach and surgical specialty. Older patients were more likely to undergo ovarian-conserving (14 \pm 3 vs. $12 \pm 5 ; P=<0.001)$ and minimally invasive procedures $(P=0.046)$. The average age of patients operated on by gynecologists was older than those operated on by surgeon group ( $15 \pm 3$ years vs. $12 \pm 5$ years; $P=<0.001$ ). The ovarian conservation group had a larger mean BMI than the oophorectomy group ( $27 \pm 8$ vs. $24 \pm 8 ; P=0.016$ ). However, BMI did not associate with surgical approach or differ between the two surgical specialty groups. Race was not found to correlate with ovarian conservation or surgical route. A post-menarchal patient was more likely to be operated on by a gynecologist $(P=0.005)$ with ovarian conservation $(P=0.005)$ via a minimally invasive procedure $(P=0.026)$.

Table 2 shows surgical characteristics relative to ovarian conservation, surgical approach, and surgical specialty. A larger adnexal mass size was found in the oophorectomy group than in the ovarian conservation group $(10.2 \pm 6.4 \mathrm{~cm}$ vs. $8.2 \pm 5.0 \mathrm{~cm} ; P=0.021)$. A larger adnexal mass size was also found in the laparotomy group than in the laparoscopy group $(10.7 \pm 6.7 \mathrm{~cm}$ vs. 7.4 $\pm 4.0 \mathrm{~cm} ; P<0.0001)$. There was no difference in mass size according to the operating surgical specialty. A patient was more likely to have her ovary conserved if she underwent an emergent 
196 procedure than if she underwent a planned procedure $(P=0.011)$, and an emergent procedure was

197 more likely than a scheduled procedure to be performed laparoscopically $(P=0.005)$. There was

198 no association between operating surgical specialty and timing of surgery (scheduled versus

199 emergent). Ovarian conservation occurred more frequently when the surgical approach was

200 laparoscopic than by laparotomy $(70 \%$ vs. $30 \% ; P<0.0001)$. The rate of conversion from

201 minimally invasive to laparotomy was the same for surgeons and gynecologists (14\%) and did not affect ovarian conservation rates.

Gynecologists were more likely to conserve the ovary than were surgeons ( $80 \%$ vs. $63 \%$;

$P<0.0001$, cOR 2.28; CI 1.16-4.48). This association remained after adjusting for potential

0.88-3.84). Gynecologists performed laparoscopic surgery $50 \%$ of the time, whereas surgeons did so $62 \%$ of the time (aOR 0.43, CI 0.22-0.85; Table 3 ).

Figure 2 shows trends of ovarian conservation and laparoscopic approach over time among gynecologists and surgeons. Over the 11-year time span, gynecologists increasingly conserved the ovaries and performed laparoscopic surgeries. Surgeons performed ovarianconserving and laparoscopic approaches at a uniform rate over the 11 years.

\section{Discussion}

In our review of 194 benign cases, the ovary was conserved $70 \%$ of the time, which is higher than published rates between $40 \%$ and $60 \%{ }^{2-4,8}$. In addition, we found that surgical cases performed emergently and laparoscopically were more likely to conserve the ovary than

217 scheduled cases and those performed by laparotomy. Forty-three out of the 194 cases (22\%)

218 were intra-operatively diagnosed as ovarian torsion. Recent data are encouraging surgeons to de219 torse adnexa and not remove adnexa, despite their ischemic appearance in young women ${ }^{11-13}$. For 
example, Santos et al. performed pelvic ultrasound follow up on women after detorsed ovaries were left in-situ even if they appeared necrotic and found that $97 \%$ of patients had multiple ovarian follicles on the affected side with no adverse outcomes ${ }^{13}$. Additionally, theoretical harms such as thrombosis have not been shown to occur ${ }^{15}$. However, oophorectomy continues to be performed in $54 \%$ to $62.5 \%$ of torsion cases c $^{2,8,12,13}$.

The malignancy rate in our cohort was $7 \%$, which is similar to that in the general population $^{2-4}$. Preoperative ultrasound can help identify benign versus malignant masses. A recent retrospective study determined that benign masses can be predicted with $100 \%$ accuracy if the mass is less than or equal to $8 \mathrm{~cm}$ and lacks complexity ${ }^{5}$. When a mass has a low probability of malignancy, the surgeon can use a minimally invasive approach and conserve the ovarian tissue.

Like other studies, ours shows that older patients, especially post-menarchal girls, are more likely than younger, pre-menarchal patients to have their surgery performed by a gynecologist. One explanation for this is that post-menarchal patients are more likely than younger patients to have established care with a gynecologist ${ }^{2,4,8}$. At our tertiary hospital, a pediatric gynecologist is available at all times. The attending physician in the pediatric emergency room decides which service to consult. Because these patients often present with right lower quadrant pain, a workup for appendicitis is often initiated, which usually includes consultation with a pediatric surgeon. Therefore, when adnexal pathology is found by imaging, the pediatric surgeon often continues to be involved in the patient's care.

Data on menarchal status was missing for $14 \%$ of the patients in our sample. We argue that it is imperative to record the last menstrual period whenever a young woman presents with abdominal pain and a mass. For instance, a physiologic follicle should be high on a physician's 
Bergeron et al

243 differential at mid cycle when one would expect ovulation. Seventy-three (37.6\%) of the

244 pathology specimens in our sample were physiologic cysts without evidence of pathologic

245 abnormality. Surgical specimens removed by surgeons were twice as likely as those removed by

246 gynecologists to be physiologic cysts. We speculate this is because gynecologists are more

247 familiar with adnexal pathology, ovarian physiology and the spontaneous regression of

248 functional cysts, making them more likely to choose conservative management instead of 249 surgery.

We found that gynecologists were less likely to perform laparoscopic surgery than

251 surgeons, and this difference persisted after results were adjusted for confounders. This finding

252 differs from those in similar published studies reporting that gynecologists were more likely than

253 surgeons to perform surgery laparoscopically ${ }^{3-5}$. However, over the 11-year time span,

254 gynecologists increasingly performed laparoscopic procedures at our institution (Figure 2). In

255 addition, some of the operative reports documented a mini-laparotomy. Compared to a typical

256 laparotomy, a mini-laparotomy incision can have improved outcomes such as reduced pain,

257 improved cosmesis and ability to perform same-day surgery. However, because we could not

258 determine the length of the mini-laparotomy incision (which varies by surgeon) from the

259 operative reports, we were unable to include mini-laparotomies as minimally invasive

260 procedures.

Our study has several limitations. First it is retrospective. Second, although our inclusion

262 criteria were broad to limit selection bias, we could have missed patients if ICD-9 and CPT

263 codes did not match or were not coded properly. Third, we adjusted for potential confounders in

264 this observational study but could not control for all possible confounders. Finally, we included

265 data from two hospitals at a single academic institution where pediatric surgeons and pediatric 
adolescent gynecologists are available full-time; thus, our results may not be generalizable to other institutions.

In conclusion, our study suggests that gynecologists are more likely than surgeons to

fertility preservation whenever possible.

\section{$\underline{\text { References }}$}

[1] Allen L, Fleming N, Strickland J: Adnexal masses in the neonate, child, and adolescent. In: Clinical Pediatric and Adolescent Gynecology. Edited by Sanfillipo J et al. New York, Informa Healthcare, 2008, pp 422-428

[2] Eskander RN, Bristow RE, Saenz NC, et al: A retrospective review of the effect of surgeon specialty on the management of 190 benign and malignant pediatric and adolescent adnexal masses. J Pediatr Adolesc Gynecol $2011 ; 24: 282$

[3] Berger-Chen S, Herzog TJ, Lewin SN, et al: Access to conservative surgical therapy for adolescents with benign ovarian masses. Obsetet Gynecol 2012; 119:270

[4] Bristow RE, Nugent AC, Zahurak ML, et al: Impact of surgeon specialty on ovarian-conserving surgery in young females with an adnexal mass. J Adolesc Health 2006; 39:411

[5] Rogers, EM, Casadiego, CG, Lacy J, et al: Preoperative Risk Stratification of Adnexal Masses: Can we predict the optimal surgical management? J Pediatr Adolesc Gynecol. 2014; 27:125

[6] Amies Oelschlager AME, Gow KW, Morse CB, et al: Management of Large Ovarian Neoplasms in Pediatric and Adolescent Females. J Pediatr Adolesc Gynecol 2016; 29:88

[7] Pfeifer S, Gosman G: Evaluation of adnexal masses in adolescents. Pediatr Clin North Am 1999; $46: 573$

[8] Hermans, AJ, Kluivers, KB, Wijen, MH, et al: Diagnosis and Treatment of adnexal masses in children and adolescents. Obstet Gynecol 2015; 125:611

[9] Seckin B, Ozdener T, Tapisiz OL, et al: Laparoscopic treatment of ovarian cysts in adolescents and young adults. J Pediatr Adolesc Gynecol 2011; 24:300

[10] Savasi I, Lacy JA, Gerstle JT, et al: Management of ovarian dermoid cysts in the pediatric and adolescent population. J Pediatr Adolesc Gynecol 2009; 22: 360

[11] Parelkar SV, Mundada D, Sanghvi, BV, et al: Should the ovary always be conserved in torsion? A tertiary care institute experience. J Pediat Surg 2014; 49: 465

[12] Cass DL, Hawkins E, Brandt ML, et al: Surgery for ovarian masses in infants, children, and adolescents: 102 consecutive patients treated in a 15-year period. J Pediatr Surg 2001; 36:693 
Bergeron et al

311 [13] Aziz D, Davis V, Allen L, et al: Ovarian torsion in children: is oophorectomy necessary? J Pediatr Surg 2004; $31239: 750$

[14] Santos XM, Cass DL, Dietrich JE: Outcome following detorsion of torsed adnexa in children. J Pediat Adolesc Gynecol 2015; 28:136

318

[15] McGovern PG, Noah R, Koenigsberg R, et al: Adnexal torsion and pulmonary embolism: case report and review of the literature. Obstet Gynecol Surv 1999; 54:601 
Table 1: Patient demographics relative to ovarian conservation, surgical approach, and surgical specialty

Ovarian conservation

Patient
Demographic

Ovarian

Sparing

$\mathrm{N}=135$

Oophor-

ectomy

$\mathrm{N}=59$

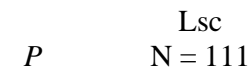

LAP

$14 \pm 3$

$12 \pm 5$

$<0.001$

$14 \pm 4$

0.016

$26 \pm 8$

$13 \pm 5$

$25 \pm 9$

$27 \pm 7$

Surgical specialty

Race

AA

43 (32)

$22(37)$

$32(29)$

$33(40)$

$25(34)$

$40(33)$
Caucasian

89 (66)

$37(63)$

$1(<1)$

$1(<1)$

$2(1)$

2(1)

0.628

Hispanic

Other

Menarchal

status $^{\mathrm{a}}$

Pre

Post

$23(17)$

$20(34)$

$93(70)$

29 (49)

77 (70)

$0(0)$

$2(2)$

$0(0)$

0.151

48 (65)

78 (65)

$1(1) \quad 0(0)$

$0(0)$

$2(2)$

Data are presented as mean and standard deviation or number and percent. Abbreviations: AA, African American; Lsc, laparoscopy; Lap, laparotomy; Gyn, gynecologist; Sur, surgeon ${ }^{\mathrm{a}}$ Menarchal status was unknown in $14 \%$ 
Table 2: Surgical characteristics relative to ovarian conservation, surgical approach, and surgical specialty

Ovarian conservation

Surgical
Characteri
stics
Mass size

$(\mathrm{cm})$

Surgery

type

$\begin{array}{lllllrr}\text { Scheduled } & 85(63) & 48(81) & & 67(60) & 66(80) & 52(70) \\ \text { Emergent } & 50(37) & 11(19) & & 0.011(68) & 0.005 \\ \end{array}$

Surgical

approach

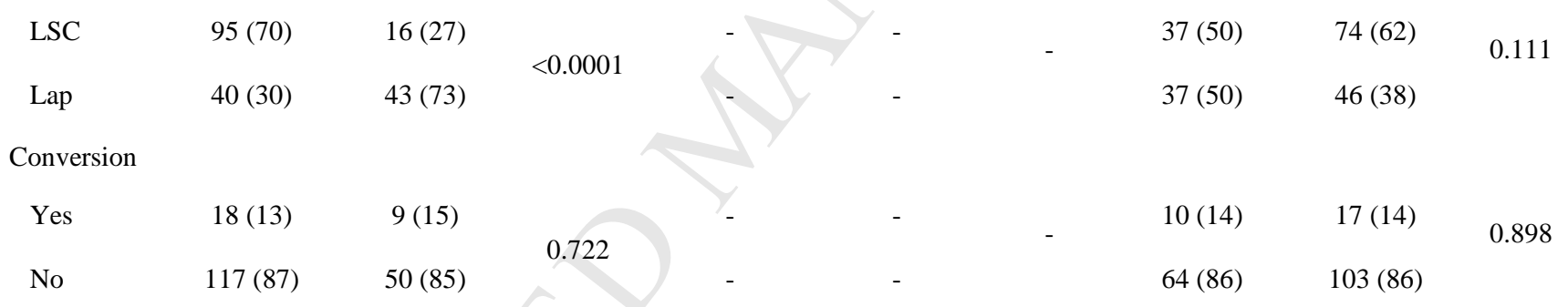

Data are presented as mean and standard deviation or number and percent. Abbreviations: Lsc, laparoscopy; Lap, laparotomy; Gyn, gynecologist; Sur, surgeon

Surgical specialty

\begin{tabular}{|c|c|c|c|c|c|c|c|c|}
\hline $\begin{array}{l}\text { Ovarian } \\
\text { Sparing } \\
\mathrm{N}=135\end{array}$ & $\begin{array}{l}\text { Oophor- } \\
\text { ectomy } \\
\mathrm{N}=59\end{array}$ & $P$ & $\begin{array}{c}\text { Lsc } \\
\mathrm{N}=111\end{array}$ & $\begin{array}{c}\text { Lap } \\
\mathrm{N}=83\end{array}$ & $P$ & $\begin{array}{c}\text { Gyn } \\
\mathrm{N}=74\end{array}$ & $\begin{array}{c}\text { Sur } \\
\mathrm{N}=120\end{array}$ & $P$ \\
\hline $8.2 \pm 5.0$ & $10.2 \pm 6.4$ & 0.021 & $7.4 \pm 4.0$ & $10.7 \pm 6.7$ & $<0.0001$ & $8.8 \pm 5$ & $8.8 \pm 6$ & 0.969 \\
\hline
\end{tabular}

$8.2 \pm 5.0 \quad 10.2 \pm 6.4$

0.021

$17(20)$

$22(30)$

0.686

(32) 
Table 3: Multivariate analysis of ovarian conservation and laparoscopy approach by surgeon and patient/surgical characteristics

\begin{tabular}{llc}
$\begin{array}{ll}\text { Characteristics } \\
\text { Gynecologist }\end{array}$ & $\begin{array}{c}\text { Ovarian Sparing } \\
\text { aOR }(95 \% \mathrm{CI})\end{array}$ & $\begin{array}{c}\text { Laparoscopy } \\
\text { aOR }(95 \% \mathrm{CI})\end{array}$ \\
Age & $1.84(0.88,3.84)$ & $0.43(0.22,0.85)$ \\
BMI & $1.08(0.99,1.18)$ & $1.09(1.00,1.19)$ \\
Mass size & $1.04(0.99,1.09)$ & $1.04(0.99,1.09)$ \\
Scheduled Surgery & $0.50(0.23,1.09)$ & $0.85(0.79,0.92)$ \\
\multicolumn{2}{l}{$\begin{array}{l}\text { Abbreviations: aOR, adjusted odds ratio; CI, Confidence interval } \\
\text { a Reference group: Surgeons }\end{array}$} & $0.50(0.25,1.02)$
\end{tabular}




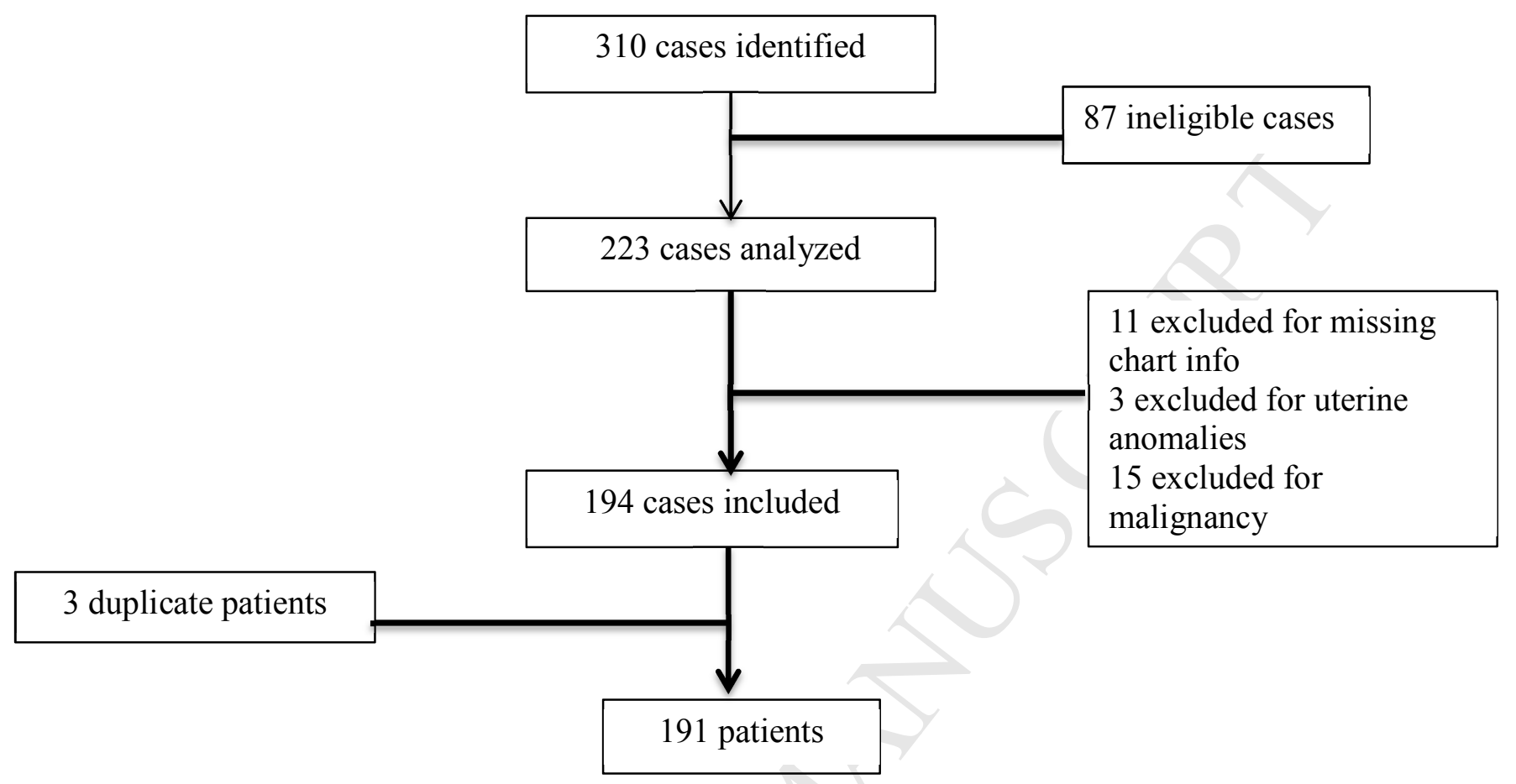


I: Title

Figure 1: Exclusion flowchart 


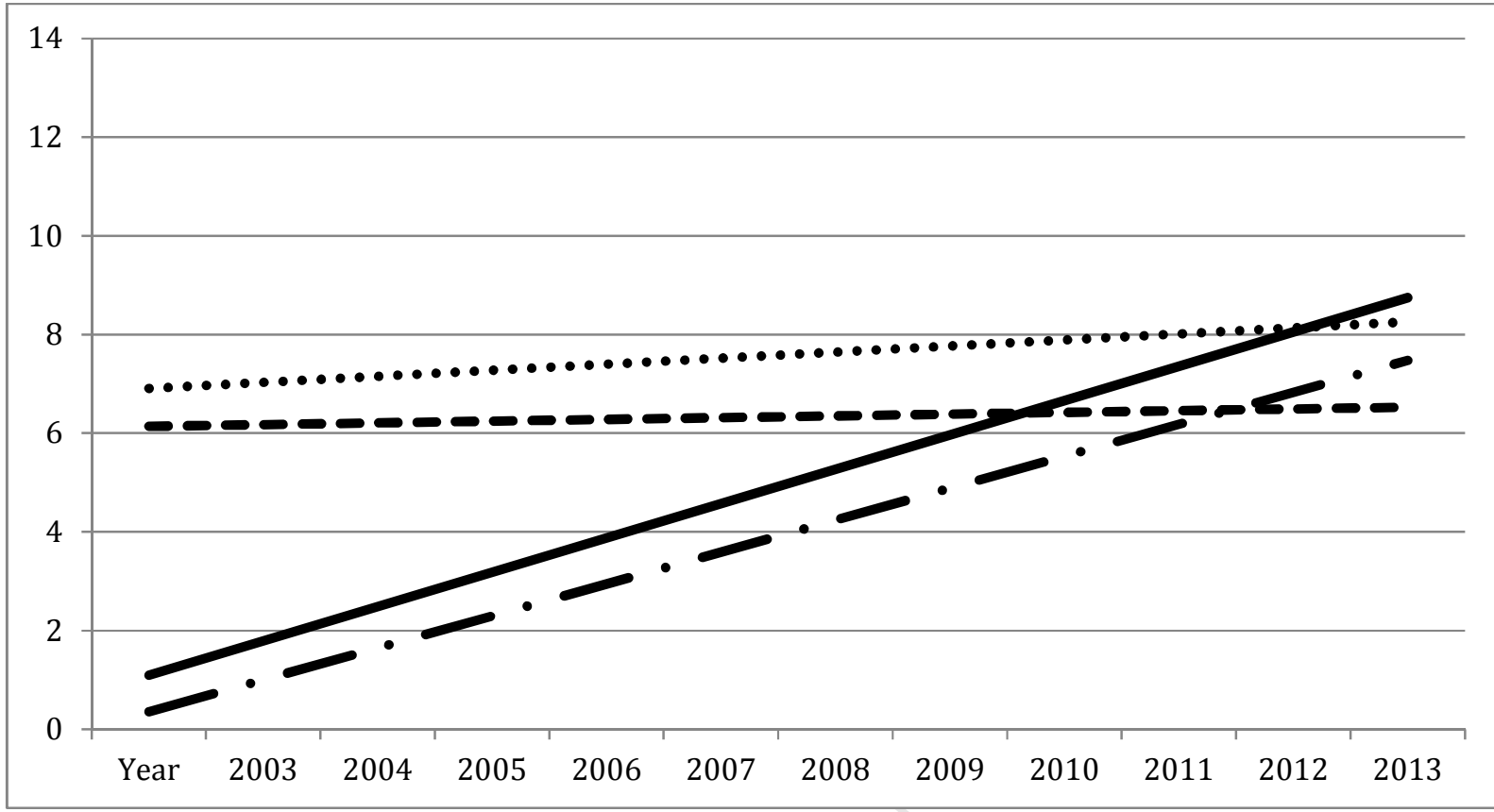


III: Title

Figure 3: Trend by Year, Ovarian Conservation and Surgical Approach by Surgical Specialty

\section{Legend}

Y-axis, number of cases; X-axis, year

Lines are trend-lines (R squared values), not data points

GYN ovarian conservation, solid line; GYN laparoscopic, dash-dotted line

Surgeon ovarian conservation, dashed line; Surgeon laparoscopic, dotted line 